\title{
REM sleep behavior disorder rhythms
}

\author{
Muna Irfan ${ }^{* *}$ and Carlos H Schenck ${ }^{2}$ \\ ${ }^{1}$ Department of Neurology, Minnesota Regional Sleep Disorders Center, Hennepin County Medical Center, Minneapolis, and Department of Neurology, University \\ of Minnesota. Minneapolis USA \\ ${ }^{2}$ Department of Psychiatry, Minnesota Regional Sleep Disorders Center, University of Minnesota, Hennepin County Medical Center, Minneapolis, USA
}

\begin{abstract}
REM sleep behavior disorder (RBD) is an intriguing parasomnia that is characterized by repeated dream enactment associated with increased muscle tone and twitch burst activity in REM sleep. This article explores a unique perspective on the relationship of RBD with various neurophysiological rhythms. We describe the clinical and polysomnographic characteristics of this disorder with diagnostic considerations. Arousal disorders simulating RBD, including Non-REM Sleep Arousal Parasomnias, Obstructive Sleep Apnea and Periodic Limb Movement Disorder, are also described. The pathophysiological basis is discussed with reference to the physiological substrates of RBD subtypes. Periodic Leg Movements associated with RBD, Rhythmic Masticatory Muscle Activity (Sleep Bruxism) and Oromandibular Myoclonus Associated with RBD, Rhythmic Movement Disorder associated with RBD, interface of RBD with epileptic abnormalities, and sleep spindles are also elaborated upon.
\end{abstract}

\begin{abstract}
Abbreviations: RBD: REM sleep behavior disorder; REM, Rapid eye movement; NREM, Non Rapid eye movement; PLM, Periodic limb movement; RMD, Rhythmic movement disorder; EEG, electroencephalogram; EOG, Electrooculogram; EMG: Electromyogram; PSG: Polysomnogram; PGO: Ponto-geniculo-occipital; PC: precoeruleus; SLD: Sublaterodorsal nucleus; SLD: Sublaterodorsal nucleus; VLPO: Ventrolateral preoptic nucleus; LC: Locus Coeruleus; LDTN: Laterodorsal Tegmental Nucleus; PPN: Pedunculopontine Nucleus; RN: Raphe Nucleus; vl-PAG: Ventrolateral periaqueductal grey; LPT: Lateral Pontine tegmentum; PD: Parkinson's disease; DLB: Dementia with Lewy Body; MSA: Multiple System Atrophy; IEA: interictal epileptiform abnormalities; iRBD: Idiopathic RBD; NC; Narcolepsy-Cataplexy; SubCD: subcoeruleus nucleus dorsalis; RAS: Reticular Activating System; SRED: Sleep Related Eating Disorder
\end{abstract}

\section{REM sleep in relation to ultradian sleep rhythms}

Sleep occurs in ultradian cycles of Non- Rapid Eye Movement (NREM) sleep followed by Rapid Eye Movement sleep, ranging from 90-120 minutes, alternating throughout the night. Typically, it comprises of 3-5 cycles per night. The duration of later REM sleep periods is longer than those noted in the earlier half of the night. REM sleep density, which is a measure of frequency of rapid eye movements in REM sleep, is also higher in the last third of the night. REM sleep has been hypothesized to be involved in memory consolidation through processing between the hippocampus and neocortex [1]. Neurophysiological monitoring of sleep requires recording of the electroencephalogram (EEG), electrooculogram (EOG) and electromyogram (EMG) [2].

\section{Polysomnographic characteristics of normal REM sleep}

The REM stage of sleep is normally characterized by low voltage intermixed frequency EEG waves, rapid eye movements, and markedly decreased skeletal muscle tone [2]. REM sleep is also called paradoxical sleep where EEG is desynchronized. On polysomnographic (PSG) monitoring, REMs are characterized as irregular sharp conjugate eye movements with initial duration $<500 \mathrm{~ms}$. Saw-tooth waves form a distinctive pattern occurring in REM sleep, which are temporally associated with REMs. They are triangular waves with frequency of 2-6 $\mathrm{Hz}$ with maximum amplitude in central leads [2]. An example of a 30sec epoch of REM sleep is shown in Figure 1A.

In feline models, ponto-geniculo-occipital (PGO) spikes have been described to originate in the pons, propagate to the lateral geniculate nucleus, and then are observed in the occipital cortex. It is hypothesized that they are related to saw-tooth waves as PGO bursts occur with high probability in relation to REMs [3]. Clusters of PGO-like subthalamic waves occurring before or during REMs have also been recorded in humans, associated with enhancement of fast oscillatory subthalamic activity [4]. PGO waves have been conventionally regarded as neurophysiological correlates of dreaming [4].

\section{REM sleep behavior disorder}

REM Sleep Behavior disorder is a distinct REM sleep parasomnia, comprising of recurrent dream enactment behavior (DEB) facilitated by abnormal loss of physiological muscle atonia, manifested as abnormal motor activity and vocalizations $[2,5,6]$.

Clinical presentation of $\mathrm{RBD}$ is characterized by abnormal vocalizations and motoric behavior representing dream enactment. Vocalization can include shouting, hollering, and cursing, suggesting

Correspondence to: Muna Irfan, Department of Neurology, Minnesota Regional Sleep Disorders Center and Department of Neurology, University of Minnesota, Hennepin County Medical Center, Minneapolis, USA, E-mail: irfan007@umn.edu

Key words: RBD, REM sleep parasomnia, rhythm, REM sleep behavior disorder periodic limb movement disorder, sleep spindles, rhythmic movement disorder, PLM in REM, pseudo-RBD

Received: October 29, 2017; Accepted: December 01, 2017; Published: December 05, 2017 


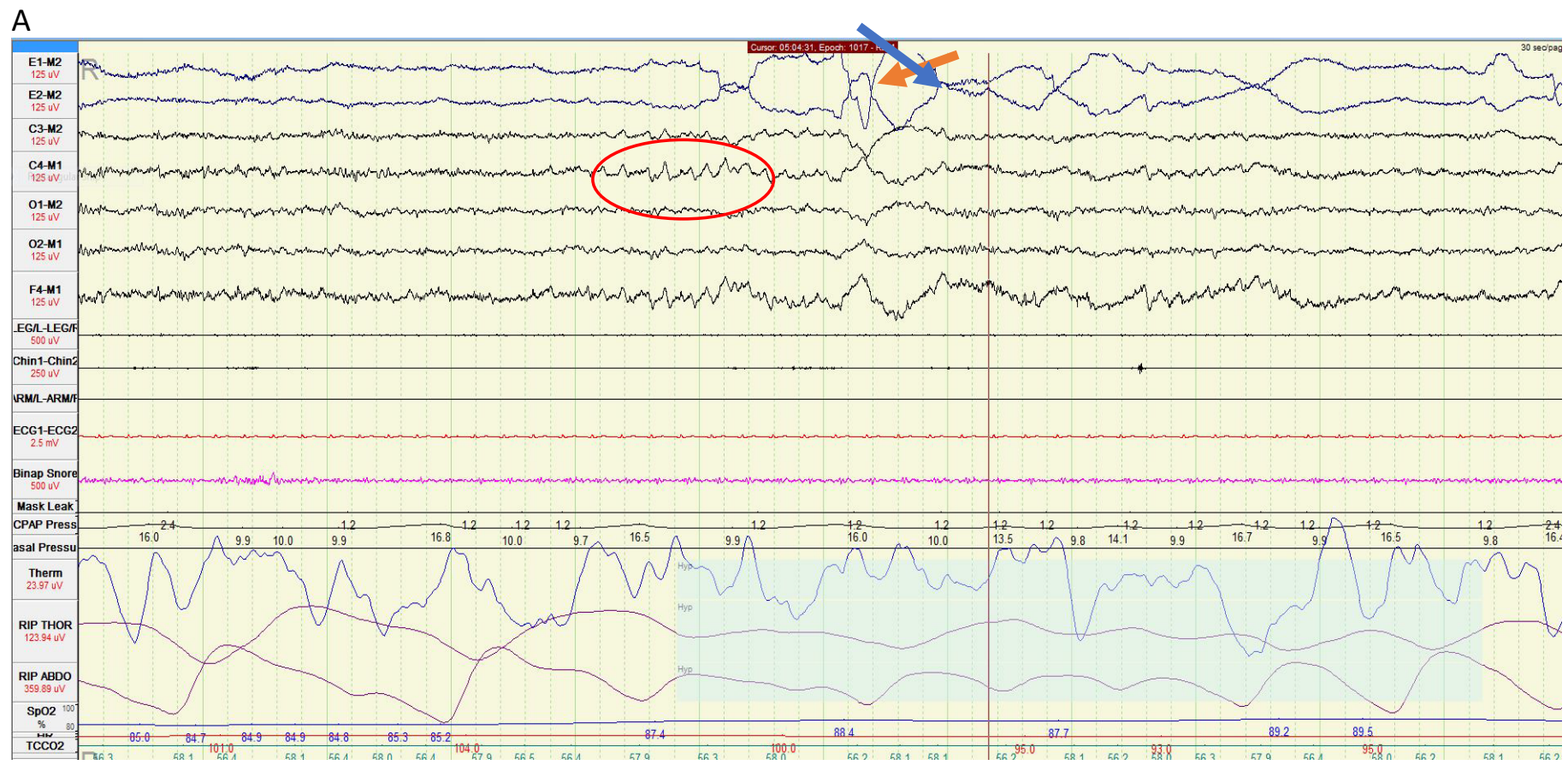

B

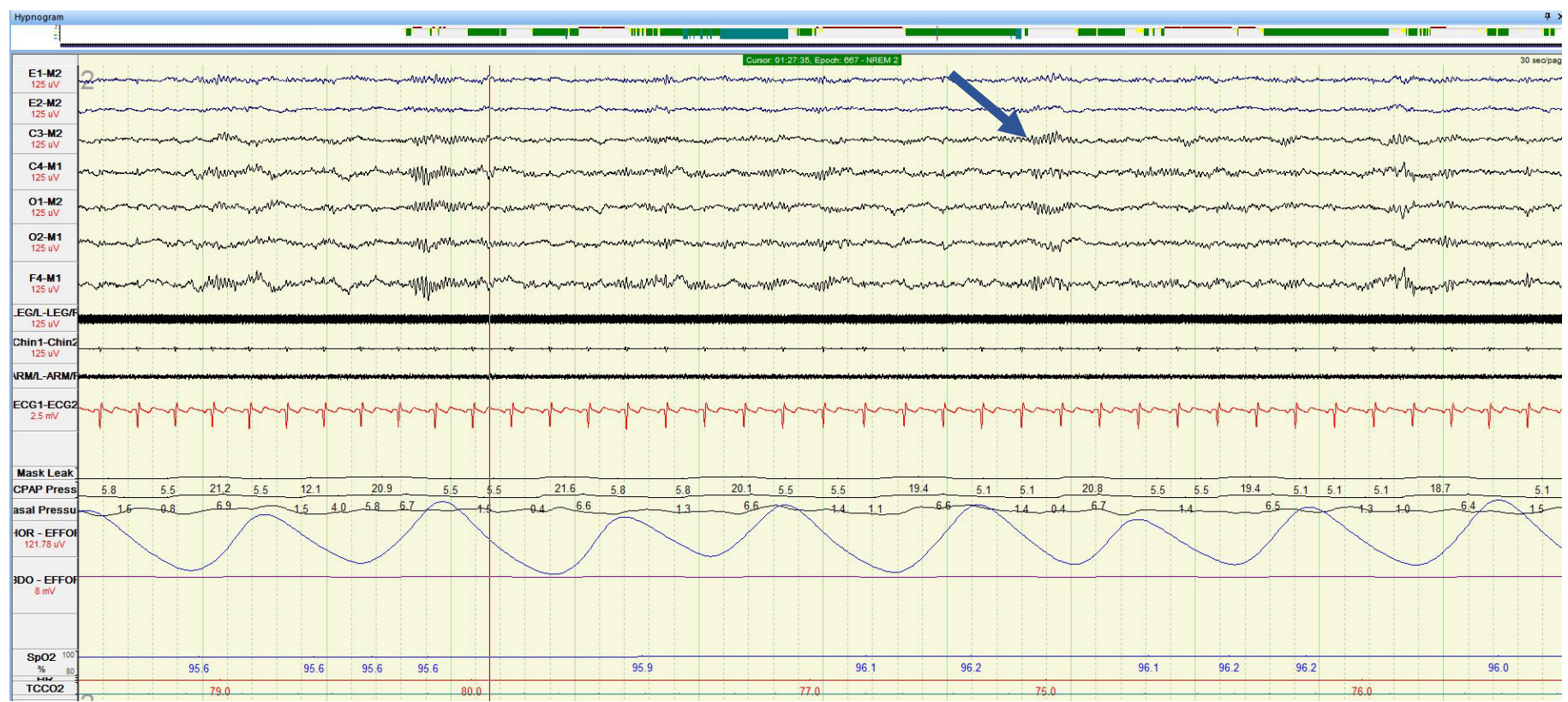

Figure 1. A) Polysomnographic recording of normal REM sleep in a 30 second Epoch. Saw tooth waves are encircled in red. Orange arrow is pointing at rapid eye movements and blue arrow demonstrates suppressed chin EMG tone. B) A 30 second Epoch demonstrates sleep spindles most prominent in central leads, denoted by blue arrow recorded in N2 sleep stage.

the disagreeable nature of the associated dreams. Motor activity can range from subtle limb movements to flailing, kicking, punching, thrashing, and jumping off the bed, resulting in injuries to patient or bed partner [6]. The dream content is typically of hostile connotation, where the patient assumes the role of defender from animals or people chasing or attacking. These episodes are typically brief with abrupt transition to alertness upon arousal. Patients often can recall vivid and elaborate details of their nightmares, the frequency of which can vary from several per night to once a month or even less frequently. According to the International Classification of Sleep Disorders, 3rd Edition, the diagnostic criteria of RBD are listed in Table 1 [2].
Table 1. ICSD 3 Criteria for Diagnosis of RBD.

\section{Diagnostic Criteria for RBD}

1 Recurrent episodes of sleep-related vocalization and/or complex motor behaviors.

These behaviors are documented by polysomnography to occur during REM sleep or presumed to occur so based on clinical history

3 PSG demonstrates REM sleep without atonia

4 Sleep related disturbance is not explained by another sleep or mental disorder, medication or substance abuse 


\section{Polysomnographic characteristics of RBD}

Physiological recording of RBD is characterized by excessive phasic or tonic activity in various EMG leads.

\section{Tonic activity}

It is described as sustained muscle activity noted in the chin musculature. According to ICSD3, it is characterized by increased muscle tone with amplitude greater than NREM activity, and lasting greater than $50 \%$ of a 30 second epoch. Figure $2 \mathrm{~A}$ demonstrates a PSG segment with excessive tonic activity in a 30 second Epoch [2].

\section{Phasic activity}

Transient muscle activity is identified in submental and limb leads or a combination of both. It is typically manifested in a 30 second epoch divided into 10 sequential, 3 second mini-epochs, with at least 5 ( $50 \%$ of total Epoch) mini-epochs exhibiting excessive bursts of transient muscle activity. These bursts are 0.1-0.5 seconds long and have amplitude at least 4 times greater than baseline EMG [2]. Neurophysiological recording of increased phasic tone is shown in Figure 2B.

\section{Pathophysiology}

\section{Neuroanatomical control of REM sleep}

REM sleep motor atonia is controlled by numerous pathways mostly localized in pons. The key structures include the "REM-on" region, comprising of precoeruleus (PC) and Sublaterodorsal nucleus (SLD). The extended part of the ventrolateral preoptic nucleus (eVLPO), Locus Coeruleus (LC), Laterodorsal Tegmental Nucleus (LDTN), Pedunculopontine Nucleus (PPN), and Raphe Nucleus (RN) are also involved. REM sleep has been suggested to be terminated by facilitation of a "REM-off" region comprising of the ventrolateral part of the periaqeductal grey matter (vl-PAG) and Lateral Pontine tegmentum (LPT) [5]. Figure $3 \mathrm{~A}$ shows the simplified circuitry where the axons originating in the "REM-on Region" synapse on the nucleus magnocellularis of the medial medulla. These axons release neurotransmitters that cause postsynaptic inhibition resulting in skeletal muscle atonia.

\section{Pathogenesis of RBD}

In typical RBD, the loss of REM sleep tone is postulated to arise from dysfunction of the above-mentioned "REM-on and off" switch. The pathway is illustrated in Figure 3B which shows disruption of axons arising from magnocellularis nucleus to the anterior horn cells, thus reducing the post synaptic inhibition of skeletal muscles, eventually leading to an increase in muscle tone. The actual neuronal circuitry is rather complex and worthy of further analytical studies. It is well recognized that a variety of conditions such as other sleep disorders, drugs, structural lesions, and neurodegenerative processes have been demonstrated to lead to RBD [6]. In RBD with narcolepsy, altered/ reduced function of hypocretinergic pathways originating in the lateral hypothalamus leads to REM sleep-waking instability causing REM sleep intrusion with excessive motor activity [32]. Various conditions have been associated with RBD as depicted in Table 2.

The presence of RBD has a strong association with the future emergence of neurodegenerative conditions, i.e alpha synucleinopathies in $81-91 \%$ of cases $[8,9]$. These include Parkinson's disease (PD), Dementia with Lewy Bodies (DLB), and Multiple System Atrophy (MSA). Table 3 lists clinical and PSG findings, as well as other co- morbid behavioral, motor, and autonomic symptoms. The presence of these symptoms in various combinations can help predict eventual phenoconversion to one of the alpha-synuclein disorders [5].

\section{Physiological substrates of RBD Subtypes}

During the past 10 years, two of the major discoveries on the control of waking and sleep were made by Garcia-Rill and colleagues that have helped revolutionize our understanding of these two fundamental states of being, with likely relevance to RBD and its subtypes. Their research was directed at the partly cholinergic pedunculopontine nucleus (PPN), the portion of the reticular activating system (RAS) that is most active during waking and REM sleep, and at its REM sleeprelated target, the subcoeruleus nucleus dorsalis (SubCD) [10]. The

Table 2. Conditions associated with RBD. Disorders with strongest linkage are denoted by asterisk.

\begin{tabular}{|l|l|}
\hline Neurodegenerative Disorders & Genetic Disorders \\
\hline Parkinson's disease * & Wilson's disease \\
\hline Dementia with Lewy bodies* & $\begin{array}{l}\text { Pantothenate kinase associated } \\
\text { neurodegeneration }\end{array}$ \\
\hline Multiple system atrophy * & Developmental Disorders \\
\hline Pure autonomic failure* & Moebius Syndrome \\
\hline Alzheimers disease & Smith-Magenis syndrome \\
\hline Progressive supranuclear palsy & Autism \\
\hline Guadaloupean parkinsonism & Tourette syndrome \\
\hline Frontotemporal Dementia & Structural Lesions \\
\hline Amyotrophic lateral sclerosis & Multiple sclerosis \\
\hline Spinocerebellar ataxia-3 $*$ & Astrocytoma \\
\hline Huntingtons'disease & Acoustic neuroma \\
\hline Myotonic dystrophy type 2 & Vascular malformations \\
\hline Orexin Deficiency & CNS vasculitis \\
\hline Narcolepsy & Infarct \\
\hline Autoimmune Disorders & Drugs* \\
\hline Voltage gated K Antibody encephalitis & $\begin{array}{l}\text { Tricyclic, tetracyclic antidepressants, } \\
\text { monoamine oxidase inhibitors, } \\
\text { Selective serotonin inhibitors, serotonin } \\
\text { norepinephrine reuptake inhibitors } \\
\text { Withdrawal: Alcohol, barbiturate, } \\
\text { amphetamine, cocaine }\end{array}$ \\
\hline Paraneoplastic cerebellar degenration & \\
\hline Ma-2 encephalitis & \\
\hline IgLON-5 Parasomnia & \\
\hline
\end{tabular}

Table 3. Electrophysiologic Dysrhythmias and signs and symptoms co-morbid with RBD.

\begin{tabular}{|l|l|}
\hline Clinical manifestation & $\begin{array}{l}\text { Occurs 2 hrs after sleep onset, more } \\
\text { episodes in the later half of night }\end{array}$ \\
\hline Timing & Seconds to minutes \\
\hline Duration & $\begin{array}{l}\text { Dream enacting movements/vocalizations, } \\
\text { ranging from twitches, arm flailing, leg } \\
\text { kicking to complex motor behaviors }\end{array}$ \\
\hline Recall & Variable vivid dream recall \\
\hline Gender predilection & Males $>$ female \\
\hline PSG findings & $\begin{array}{l}\text { Increased phasic and tonic EMG tone in } \\
\text { chin and limb leads during REM sleep, with } \\
\text { irregular heart and respiratory rate. }\end{array}$ \\
\hline Co-morbid autonomic dysrhythmias & $\begin{array}{l}\text { Orthostatic hypotension } \\
\text { Cardiac autonomic dysfunction: Reduced } \\
\text { heart rate and blood pressure variability } \\
\text { Constipation } \\
\text { Sexual dysfunction: Erectile dysfunction, } \\
\text { ejaculation problem }\end{array}$ \\
\hline Urinary dysfunction
\end{tabular}


A

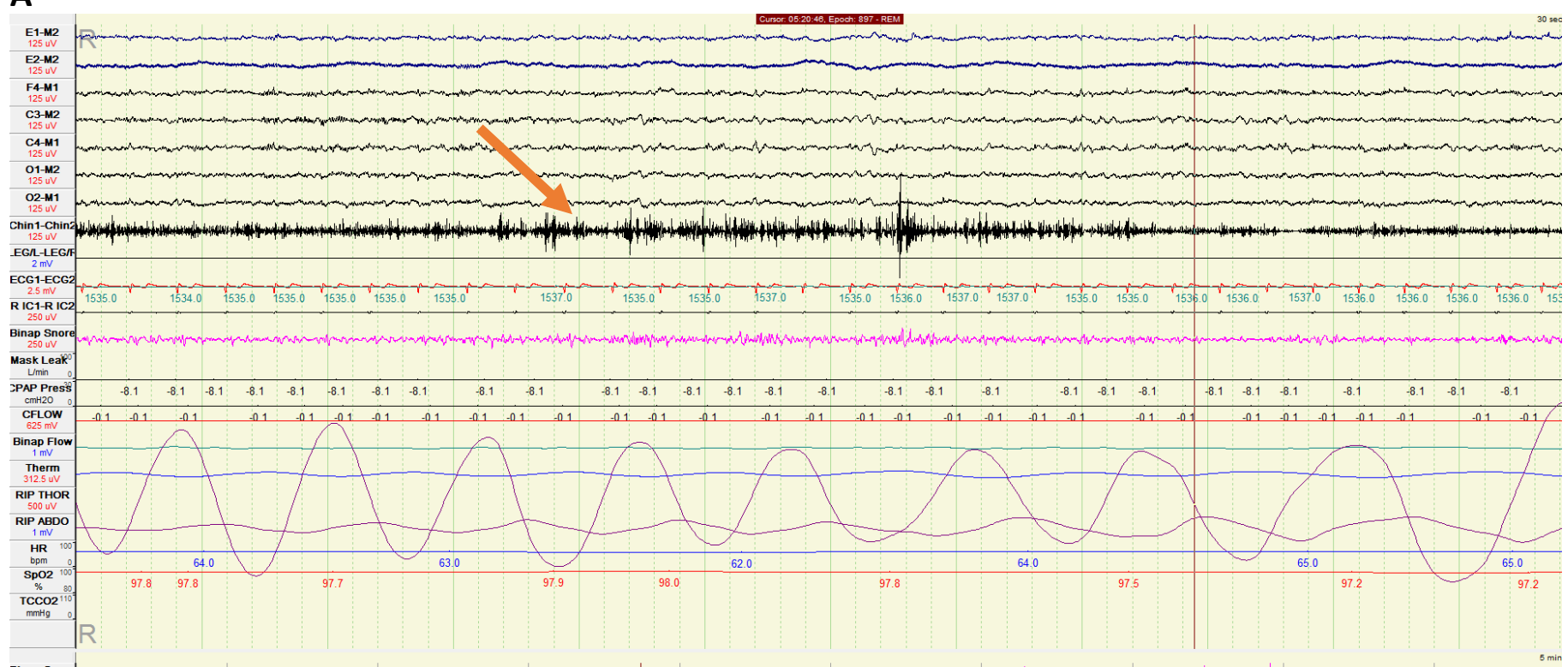

B

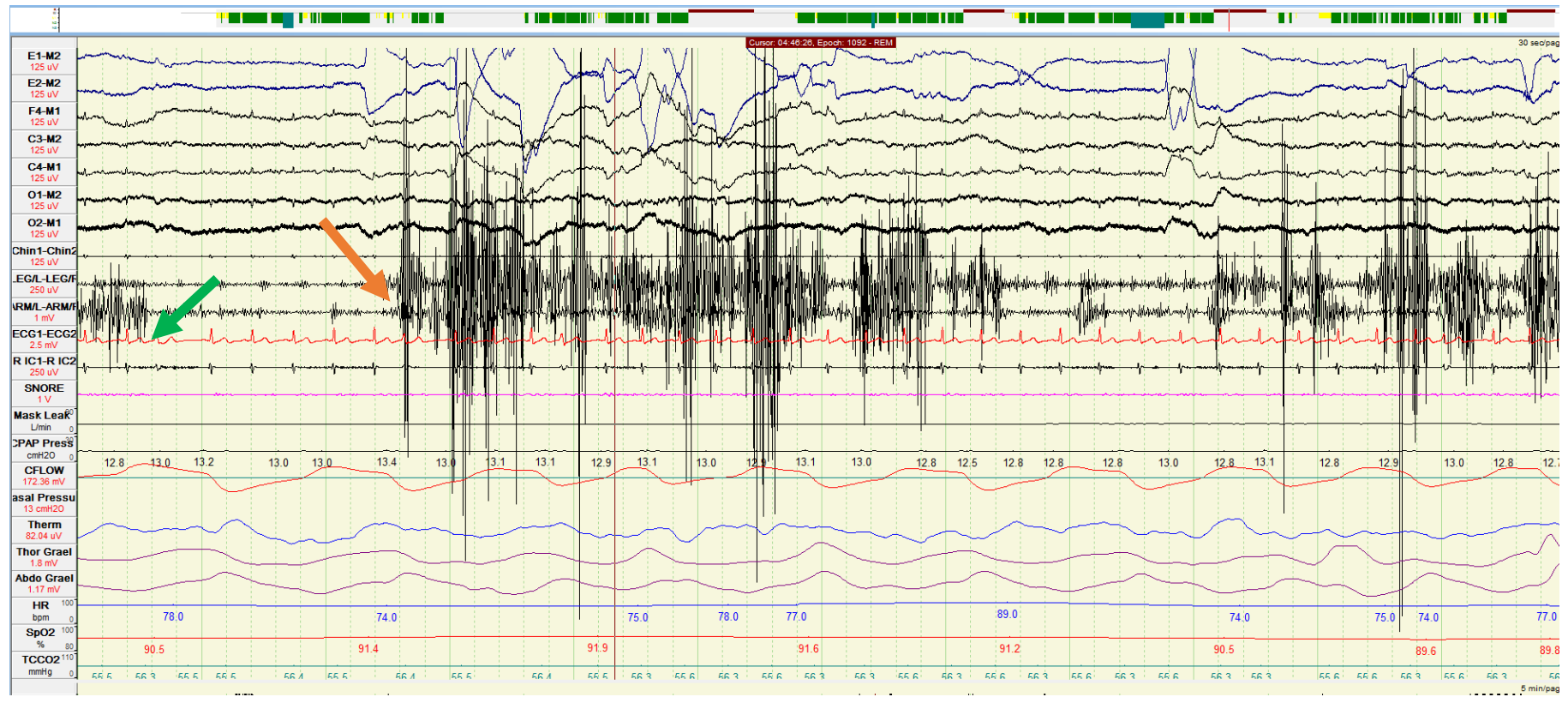

Figure 2. A) Polysomnographic recording of increased tonic activity (denoted by orange arrow) in chin EMG lead for over fifty percent of the 30 second epoch. B) Thirty-second epoch of polysomnogram showing increased phasic activity in the lower limb leads (left leg to right leg) denoted by the orange arrow, and in the upper limb leads (left arm to right arm) denoted by the green arrow.

PPN modulates the manifestation of REM sleep through descending projections to the SubCD [11]. These regions possess a proportion of cells that are electrically coupled through gap junctions (first discovery), thus promoting coherence within each nucleus; and every cell in these nuclei shows gamma band activity that can export gamma frequency activity to its targets (second discovery) [12]. Neither mechanism has yet been studied extensively for its involvement in RBD or its subtypes. Nevertheless, research into these areas should permit a deeper understanding of the pathophysiology of RBD disease processes, and perhaps point to novel therapeutic directions. These discoveries are described in detail in a recent book by Garcia-Rill [13].
The RAS is made up of the PPN, locus coeruleus (LC), and dorsal raphe nucleus, with the PPN being most active during waking and REM sleep [16]. The LC and raphe nucleus both fire during waking and somewhat during slow wave sleep, but not during REM sleep. The PPN is composed of different populations of cholinergic, glutamatergic, and GABAergic neurons [17].

PPN neurons increase firing during REM sleep ("REM-on"), or during both waking and REM sleep ("Wake/REM-on"), but decrease during slow-wave sleep (SWS) [14,16-18], suggestive of increased excitation only during activated states. Stimulation of the PPN 
A
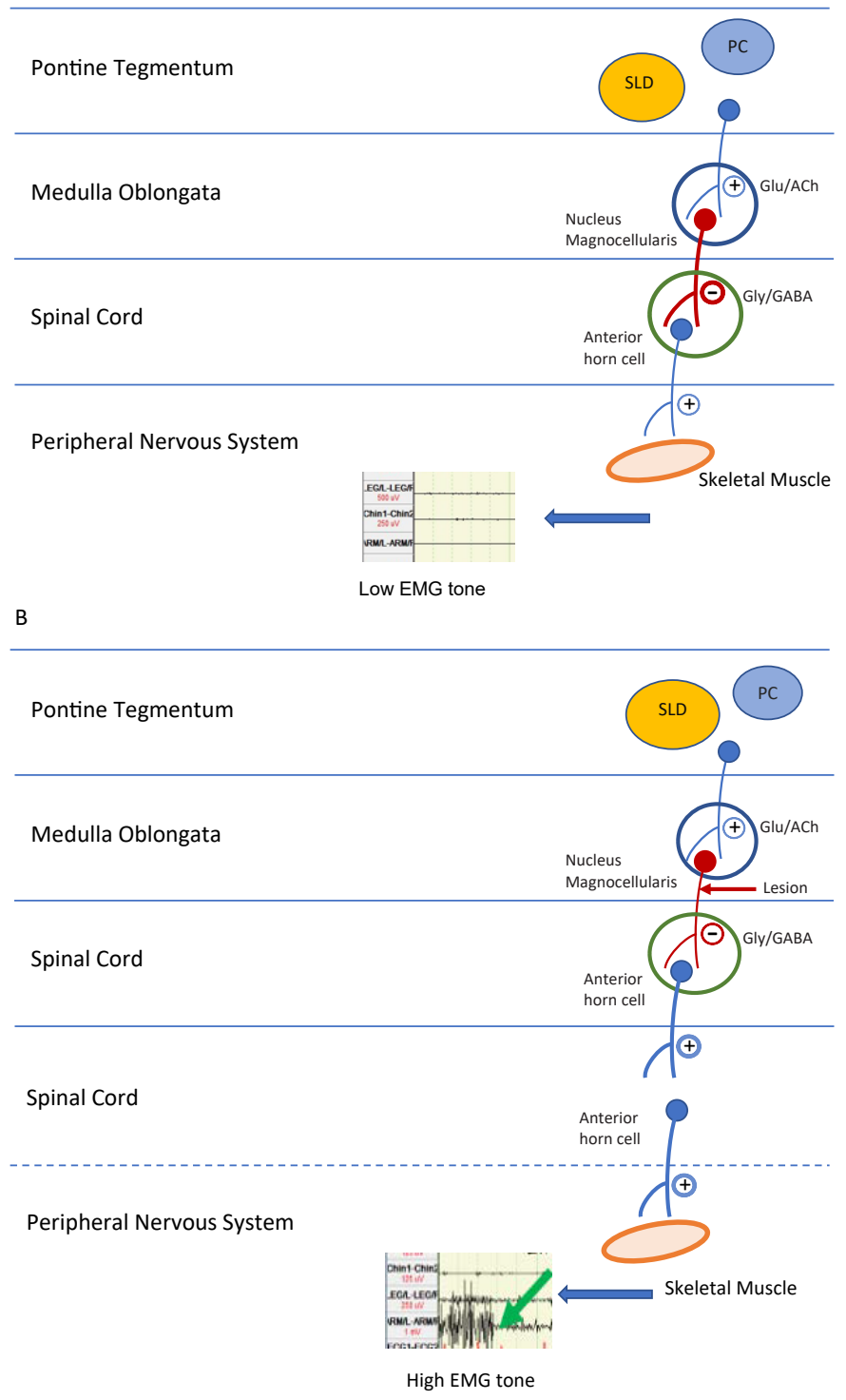

Figure 3. A) Diagrammatic representation of neural pathways mediating normal skeletal muscle atonia in REM sleep. B) Diagrammatic representation of disruption of the pathway leading to loss of REM associated atonia and thus increasing EMG tone.

Abbreviations: Glu, glutamate; ACh, acetylcholine; Gly, glycine; GABA, gamma aminobutyric acid; +, excitatory neurotransmitter; _, inhibitory neurotransmitter

potentiates the appearance of fast $(20-40 \mathrm{~Hz})$ oscillations in the cortical EEG, outlasting stimulation by 10 to 20 seconds [19]. REM sleep is distinguished from NREM sleep and wakefulness by low amplitude, high frequency EEG activity, generalized skeletal muscle atonia [20], and PGO waves in humans and cats (called P waves in the rat) [21]. Nuclei located in the pons, including the SubCD, are critical for generation of this state [22].

\section{Discovery of electrical coupling and of gamma band activity in the RAS}

Garcia-Rill and colleagues discovered that some cells in the PPN and in the SubC were electrically coupled $[23,24]$. They reported the presence of dye coupling and spikelets in some PPN and SubC neurons, as well as in the parafascicular nucleus, an ascending target of the PPN involved in thalamocortical oscillations [25]. Gamma band activity during waking has different mechanisms from gamma band activity during REM sleep. Intracellularly, protein kinase C (PKC), which modulates kainic acid receptors (and kainic acid injected in the PPN induces REM sleep), enhances $\mathrm{N}$-type channel activity and has no effect on P/Q-type channel function [26], but CaMKII, which modulates NMDA receptors (NMDA induces waking when injected into the PPN), was shown to modulate P/Q-type channel function [27]. Therefore, the two calcium channel subtypes in the PPN are modulated by different intracellular pathways, N-type by the cAMP/ PK pathway, and P/Q-type via the CaMKII pathway. Additionally, there are three cell types in the PPN, those bearing only N-type calcium channels, those with both N- and P/Q-type, and those with only P/Qtype calcium channels $[28,29]$. The implications from these results are that, i) there is a "waking" pathway mediated by CaMKII and P/Qtype channels and a "REM sleep" pathway mediated by cAMP/PK and N-type channels; and ii) different PPN cells fire during waking (those with $\mathrm{N}+\mathrm{P} / \mathrm{Q}$ and only $\mathrm{P} / \mathrm{Q}$-type) vs REM sleep (those with $\mathrm{N}+\mathrm{P} / \mathrm{Q}$ and only N-type) $[28,29]$

In regards to the cerebral cortex, the difference between gamma band activity during waking vs. REM sleep appears to be a lack of coherence [30]. Thus, brainstem driving of gamma band activity during waking carries with it coherence across distant cortical regions, while driving of gamma band activity during REM sleep does not include coherence across distant regions [30,31]. These results suggest that, i) brainstem centers drive gamma band activity that is manifested in the cortical EEG; ii) during waking, brainstem-thalamic projections include coherence across regions; and during REM sleep, which is controlled by the SubCD region (with lesion of this region eliminating REM sleep, while injection of carbachol induces REM sleep signs), gamma band activity drives cortical EEG rhythms without coherence.

\section{Implications for RBD subtypes}

Currently, very little is known about gap junctions, high threshold calcium channels, or sodium-dependent subthreshold oscillations in human RBD. However, the close relationship between NarcolepsyCataplexy (NC) and RBD (present in up to $60 \%$ of NC patients [32]) points to the involvement of the orexin/hypocretin neurons in the dorsolateral hypothalamus, which degenerate in narcolepsy. The hypothalamic orexin sites, as well as basal forebrain sites, drive waking through the RAS. Stimulation of these regions must be applied for much longer periods $(10-20 \mathrm{sec})[33,34]$ compared to RAS stimulation $(1-2 \mathrm{sec})$ to induce waking $[13,33,35]$. In addition, optogenetic studies have found that induction of waking by stimulation of orexin neurons is blocked by inactivation of the LC in the RAS [33]. Thus, it appears that the RAS may be the final output for the arousal induced by some of these modulatory regions [13]. Therefore, the co-expression of RBD with NC could well have its origin at the level of the RAS.

The literature on medication-induced RBD suggests that most classes of antidepressants can be triggers for RBD. Buproprion, a dopaminergic/noradrenergic agent, has not been reported to induce $\mathrm{RBD}$, and is known to block gap junctions, at least in the heart [36]. In contrast, SSRIs, which do induce RBD in some patients, are also thought to act somewhat through gap junction blockade [37]. Therefore, these findings suggest that gap junctions are not involved in drug-induced RBD.

Finally, idiopathic RBD in children [38], which is rare, appears to involve a lack of maturation of REM sleep atonia during the wellknown developmental decrease in REM sleep [39]. Garcia-Rill and colleagues recently reported that the expression of $\mathrm{N}$-type calcium 
channels in PPN decreases by $\sim 350 \%$ during this period while the expression of $\mathrm{P} / \mathrm{Q}$-type calcium channels decreases by a fraction of that number [40]. These findings suggest that the developmental decrease in REM sleep may be due at least in part by a decrease in the expression of N-type channels in the PPN. The role of these channels in RBD, as stated above, has not yet been studied.

\section{Future directions}

Future research should address the roles of gap junctions and electrical coupling, as well as high threshold calcium- and sodiumdependent intrinsic membrane oscillations, in RBD and its subtypes. The development of novel therapeutic, and perhaps preventive, strategies may depend on the examination of the development of REM sleep control and on the role of intracellular pathways modulating the manifestation of REM sleep vs waking. The foregoing also strongly points to the SubCD, and perhaps also the LC, as a potential target for study.

\section{Periodic leg movements (PLMs) associated with RBD}

PLMs are described as periodic repetitive movements (with a typical frequency of every 20-40 seconds), consisting of toe extension, ankle dorsiflexion, and knee and hip flexion, which depending on the severity can also be manifested in the upper extremities and the trunk. While PLMs are most commonly present in Restless Legs Syndrome (RLS), affecting approximately $80 \%$ of RLS patients [2], they can also be found in other sleep disorders, including RBD. PLMs are thought to be a manifestation of dopaminergic dysfunction. They predominantly occur in NREM sleep, although they can be noted less frequently in REM sleep as well. Various large reported series on RBD patients have found the presence of PLMs in NREM and REM sleep (with hourly indices $>10-20)$ in approximately one-half to three-quarters of RBD patients [41,42]. PLMs as abnormal rhythmic motor activity thus represent another common form of motor dyscontrol during sleep in RBD. An intriguing feature of PLMs in RBD is the lack of concomitant signs of autonomic arousal with the PLMs, in contrast to PLMs not associated with RBD where there is often EEG and other signs of arousal $[41,42]$.

The underlying mechanisms for the strong association of PLMs in $\mathrm{RBD}$ may be explained by two experimental animal models, one in cats [43] and one in rats [7]. The experimental animal model in cats involved neurotoxic lesions at the ventral mesopontine junction (VMPJ) [43]. Previous work by these authors (Lai and Siegel group) had found that the motor inhibitory system in the brainstem extends from the medulla to the VMPJ. The three groups of cats receiving neurotoxic lesions at three separate sites within the VMPJ (viz. rostrolateral, rostromedial and caudal sites) each showed a significant increase in PLMs, and an increase in isolated non-periodic leg movements in NREM sleep, along with increased tonic muscle activity in REM sleep, closely mirroring findings in human RBD. Furthermore, an increase in phasic motor activity in REM sleep, also resembling that seen in human RBD, was found in the caudal VMPJ lesioned cats. The proximity of the VMPJ region to the substantia nigra suggests that the frequent progression (in $>80 \%$ of patients in reported series $[8,44]$ ) from idiopathic RBD (iRBD) to parkinsonian disorders in humans may be mediated by the spread of synuclein-mediated damage from the VMPJ subregions identified in this study into the substantia nigra. Moreover, in the original report on the selective phenoconversion from iRBD to a parkinsonian disorder, identified in $38 \%$ of patients originally diagnosed with $\mathrm{iRBD}$ [45], the group of iRBD patients that eventually phenoconverted had a significantly increased PLM hourly index at baseline (more than double) compared to the group of iRBD patients who had not phenoconverted at the time of latest follow-up: $85.2 \pm 44.8$ vs. $35.9 \pm$ $21.1(\mathrm{p}=0.003)$. This finding suggests that a high PLM index in RBD could represent a biomarker for relatively imminent phenoconversion to parkinsonism. Additionally, approximately half of all the iRBD patients at baseline had isolated non-periodic leg movements in NREM sleep, which was also found in the cats receiving neurotoxic lesions at each of the three VMPJ sites. Therefore, the VMPJ neurotoxic lesion animal model appears to be quite relevant to the strong presence of PLMs in human RBD, and to the strong link between RBD-PLMs and parkinsonism.

In regards to the experimental rat model of PLMs-RBD [7], the impetus for pursuing this model involving the inferior colliculus (IC) was that an abnormal auditory brainstem evoked potential wave $\mathrm{V}$ had previously been reported in RBD patients [46], as well as in spinocerebellar ataxia patients [47] who also can have RBD [48-51] The external cortex of the IC (ICX) has been demonstrated not only to be involved in auditory processing, but also to participate in the modulation of motor activity. Those clues led the Lai-Siegel group to generate a hypothesis on the role of ICX in movement disorders during sleep. Rats were surgically implanted with electrodes for EEG and EMG recordings and guide cannulae were directed at the ICX for drug infusions. vPSGs were analysed to detect normal and abnormal sleep states. Baclofen, a gamma-aminobutyric acid B (GABA-B) receptor agonist, infused into the ICX increased PLMs in NREM sleep and REM sleep, and also induced tonic muscle activity in REM sleep; it also elicited RBD-like behaviors during the infusion and post-infusion period. In contrast, saclofen, a GABA-B receptor antagonist, did not produce significant changes in motor activities in sleep. The findings from this study suggested that GABA-B receptor mechanisms in the ICX may be implicated in the pathology of RBD, including the link between PLMs and RBD.

\section{Rhythmic masticatory muscle activity (RMMA: sleep bruxism) and oromandibular myoclonus (OMM) in RBD}

A study compared the RMMA typical of sleep bruxism and OMM during REM sleep in patients with iRBD, and in Parkinson disease (PD) patients with RBD (PD + RBD) [52]. PSG data were collected from 9 age-matched controls and 28 patients (mean age, $66 \pm 1.7 \mathrm{yrs}$ ) with PSG-confirmed RBD. The two patient groups included 13 patients with $\mathrm{iRBD}$ and 15 patients with PD + RBD. RMMA, a marker of sleep bruxism, and OMM were scored blind to subject's diagnosis from jaw EMG recordings during sleep. The RMMA index was significantly higher during REM sleep in iRBD subjects compared to controls $(\mathrm{p}<.01)$, and was significantly higher during NREM sleep in both iRBD and RBD + PD groups compared to controls $(\mathrm{p}<.03)$. A positive sleep laboratory diagnosis of sleep bruxism was made in $25 \%$ of $\mathrm{iRBD}$ and $\mathrm{RBD}+\mathrm{PD}$ patients. In iRBD, patients had more OMM during REM sleep than controls (2.4 times higher; $\mathrm{p}=.01$ ). Therefore, the presence of a high frequency of RMMA/sleep bruxism during REM sleep should raise suspicion for RBD and prompt further evaluation. Also, RBD patients should be questioned about sleep bruxism, with possible dental referral.

A case was reported on sleep bruxism as a manifestation of subclinical RBD [53], i.e. REM sleep without atonia with limb jerking and twitching and minor behaviors, but without complex or aggressive dreamenacting behaviors [54]. A 59-year-old man with temporomandibular joint pain and dysfunction presented to a sleep clinic with a long history of nocturnal sleep bruxism. Video-PSG documented episodes of teeth 
grinding and clenching occurring exclusively during REM sleep, which were often associated with movements of the hands and fingers and/or brief vocalization. Throughout REM sleep there was excessive phasic chin and limb EMG twitching. REM-atonia was preserved, along with preserved REM-NREM sleep cycling. Sleep bruxism in this case was concluded to be a manifestation of subclinical RBD, raising the question of whether there is a common pathophysiology with REM sleep bruxism and (subclinical) RBD.

Finally, a case was recently reported on sleep bruxism triggering recurrent sleep masturbation episodes during REM sleep (and NREM sleep) in a 49-year-old man who did not have RBD [55]. The sleep masturbation as a complex rhythmic behavior emerging during REM sleep was triggered by another rhythmic behavior in REM sleep, viz. sleep bruxism (with EEG arousal) that was documented to be severe, with a bruxism episode index of $10.1 / \mathrm{hr}$, and a bruxism burst index of $12.8 / \mathrm{hr}$. This case raises questions about the mechanisms of sleep bruxism arousal induced behavioral release in REM sleep, with only one type of behavior (sexual) being released. Sleep masturbation thus represents another category of complex behavior emerging in REM sleep that was not triggered by $\mathrm{RBD}$, but rather by sleep bruxism.

\section{Rhythmic movement disorder (RMD) associated with RBD}

RMD is classified as a Sleep Related Movement Disorder in the International Classification of Sleep Disorders, 3rd Edition [2]. RMD features repetitive movements of large muscle groups, often involving the head and neck, which typically emerge immediately during wakesleep transitions and during sleep. RMD behaviors span head banging, leg banging, body rocking, head rolling, body rolling, leg rolling, and their combinations. RMD is most commonly seen in children, especially infants, with the majority of these rhythmic movements stopping as childhood progresses. RMD persisting into adolescence is often associated with mental retardation, autism, attention deficit disorder, and other neuropsychiatric conditions. Three cases of RMD have been reported with RBD [56,57]. The first two cases were reported by Manni and Terzaghi with video-PSG capturing head-rolling that occurred during REM sleep in the context of RBD episodes, in two patients with idiopathic RBD, and without personal or familial history of sleep related RMD [56]. The authors speculated that the activation of neuronal pathways that underlie the loss of REM sleep atonia and disinhibition of motor-behavioral release in RBD may also involve the activation of brainstem Central Pattern Generator neuronal networks. This would lead to the release of rhythmic movements during RBD episodes, thereby allowing the re-emergence, in the pathological condition of RBD in later life, of a motor behavior typically seen in an early developmental stage.

The third reported case involved a 41-year-old man with childhoodonset and adult persistence of RMD, with rhythmic leg kicking during wake-sleep transitions [57]. The RMD had worsened after the onset of RBD. Video-PSG confirmed the diagnosis of RBD, with loss of REM sleep atonia and with episodes of kicking and punching during REM sleep. More than 1,000 rhythmic limb movements were recorded, with the majority occurring during wake-N1 sleep and N1 sleep-wake transitions, but rhythmic limb movements were also present in all sleep stages (including REM sleep). Periodic limb movements of sleep, often found with RBD, were absent. The RMD persisted despite successful treatment of RBD with zopiclone (after a failed trial of clonazepam). The RMD also persisted despite a trial of gabapentin. The persistent RMD prevented this patient from sleeping with his partner.

\section{Interface of RBD with epilepsy and interictal EEG ab- normalities in older adults}

RBD in elderly subjects with epilepsy has been studied for its frequency and the clinical aspects of comorbidity [58]. Eighty epilepsy patients $>60$ years old (mean age 71 years; $47 / 80$ males) were screened for RBD by clinical interview and video-PSG. Previously undiagnosed or misdiagnosed idiopathic RBD was found in $12.5 \%(10 / 80)$ of patients, all men. RBD was significantly more frequent in male patients with epilepsy compared to female epileptics. In 6 of 10 patients, RBD preceded the onset of epilepsy by a mean of 4.5 years. In 4 of 10 patients, RBD followed the onset of epilepsy by a mean of 9.7 years and was more likely to occur in patients with sleep-related seizures. The authors concluded that undiagnosed or misdiagnosed RBD can coexist with epilepsy in the elderly. This should encourage increased clinical awareness of the RBD-epilepsy comorbidity for the benefit early diagnosis and therapy for RBD.

Another study by the same authors (Manni, Terzaghi and colleagues) examined the implications of interictal epileptiform abnormalities (IEA) in idiopathic RBD (iRBD), particularly the risk of misdiagnosing RBD episodes as epileptic nocturnal seizures [59]. Thirty iRBD patients ( 28 men), mean age 66 years were studied, and $27 \%(6 / 30)$ had IEA during routine wake EEG or during video-PSG. Comparison of the patients' demographic data and RBD features found no differences between RBD with IEA and RBD without IEA. Two patients had IEA during REM sleep, and IEA was also found in NREM sleep and wakefulness. Paroxysmal nocturnal episodes during vPSG were typical RBD parasomnia behaviors rather than motor-behavioral seizure events. Therefore, IEA in RBD should be interpreted as a nonspecific finding, probably related to brain aging.

\section{Sleep spindle changes with RBD}

Sleep spindles comprise an EEG hallmark of N2 sleep, and have traditionally been classified as fluctuating $10-16 \mathrm{~Hz}$ oscillations lasting 0.5-2.0 seconds. Sleep spindles of similar morphology have been found in all studied mammalian species. The underlying mechanisms of sleep spindle generation reflect the intrinsic properties and interactions between inhibitory cells in the thalamic reticular nucleus and bursting thalamo-cortical relay neurons [60]. Evidence is mounting that human sleep spindles comprise diverse phenomena rather than a single prototypic phenomenon. It is now known that most sleep spindles are spatially restricted to specific brain regions. There is topographical organization in sleep spindle frequency, with a sharp transition around the supplementary motor area between fast $(13-15 \mathrm{~Hz})$ centroparietal spindles and slow $(9-12 \mathrm{~Hz})$ frontal spindles.

The first vPSG study to investigate spindle properties in RBD patients compared to healthy controls found that RBD patients had significantly lower sleep spindle density [61]. Further analysis of the RBD patients found that sleep spindle densities were lower for fast frequencies, but higher for slow frequencies. The commonly used frequency threshold for separating fast from slow sleep spindle frequency was $13 \mathrm{~Hz}$. Given the altered sleep spindle activity in RBD, this finding should be further investigated as another potential marker of evolving neurodegeneration in RBD patients.

Another controlled study found decreased sleep spindle density in patients with idiopathic RBD, and patients with RBD and Parkinson's disease, compared to PD patients without RBD and controls [62]. The authors commented that the lower sleep spindle density suggested involvement of pre-thalamic fibers involved in the genesis of sleep 
spindles, and they also pointed out that altered sleep spindle density could be an early biomarker of PD.

A case of an 80-year-old woman with RBD and Dementia with Lewy bodies (DLB) was reported, whose pre-treatment video-PSG study confirmed RBD, but also found the complete absence of sleep spindles, and loss of slow-wave sleep [63]. Therapy with donepezil, an anticholinesterase inhibitor, $3 \mathrm{mg}$ daily, increased to $5 \mathrm{mg}$ daily after 1 week, induced marked control of persecutory delusions, hallucinations and depression, and improved cognitive function. Repeat videoPSG during donepezil therapy found considerable sleep spindle activity. Therefore, enhanced cholinergic modulation induced by the donepezil reestablished sleep spindle activity and exerted remarkable clinical improvement of DLB symptoms. The authors postulated that the mechanism of therapeutic improvement was cholinergic modulation of the thalamo-cortical neural circuit, as manifested by the reemergence of sleep spindles that had been suppressed with untreated DLB. Therefore, changes in sleep spindle activity, morphological distribution, frequency and density in relation to RBD represent complex neuronal interplay, with important implications. For example, these sleep spindle changes can potentially assist with predicting future neurodegenerative phenoconversion, and thus merit further clinical scientific investigation.

\section{Autonomic dysrhythmias associated with RBD}

Autonomic derangement in RBD can include gastrointestinal, cardiovascular, urinary and sexual dysfunction. Orthostatic symptoms and hypotension are noted in RBD, but to a significantly higher degree in RBD with PD compared to idiopathic RBD (iRBD) [64].

In idiopathic RBD, cardiovascular autonomic function is affected in both the awake and sleep state, reflected by changes in R-R interval variation, Valsalva ratio, and heart rate response to standing on autonomic testing. During sleep, reduced tonic and phasic heart rate variability is noted. Despite the presence of cardiac autonomic symptoms in idiopathic RBD, it has not been established to predict the risk of conversion to a neurodegenerative disorder.

Constipation has been well recognized as a prodromal feature of PD [65]. A cross sectional study showed higher number of patients with iRBD to have constipation compared to controls [66], but it has low specifity and low positive predictive value, due to the high prevalence of constipation in the general population. While sexual dysfunction has been widely noted in patients with PD, there is also indication that the severity of erectile dysfunction may predict conversion from iRBD to neurodegeneration [64]. Erectile dysfunction is higher in patients with iRBD compared to controls [48], although the predictive value remains low. Urinary dysfunction has also been found to a higher degree in iRBD patient than in controls [66], and is considered a prodromal feature of PD [65].

It has been suggested based on studies that patients with phenoconversion to MSA had greater bladder/bowel dysfunction and a cardiac chronotropic response to tilt $>10$ beats/minute, while those who developed PD or DLB showed lesser responses to tilt. [67]. Thus, the presence and severity of various autonomic symptoms may assist in predicting conversion to alpha-synucleopathies.

\section{Disorders mimicking RBD}

It is imperative to diagnose RBD accurately due to the serious implication of the association with neurodegenerative disorders. Not only can this prognosis cause a substantial psychological burden, but the diagnosis also warrants regular surveillance. Treatment will also vary depending on the etiology. Other sleep related conditions can mimic RBD with dream-enactment, the most noteworthy of which are discussed below:

\section{NREM sleep parasomnia arousal disorders in adults (Sleepwalking, Sleep Terrors)}

Dream enacting behavior (DEB), including complex and elaborate $\mathrm{DEB}$, has been reported in adults with NREM sleep arousal disorders [68-71], and is recognized in the ICSD-3 as an important differential diagnosis of RBD [2].

\section{Obstructive sleep apnea (OSA)}

DEB can also occur with an underlying OSA, a condition termed as "Pseudo-RBD". In such cases, reports of flailing and kicking of limbs associated with some dream mentation were documented to arise from hypopnea and apnea induced arousals during video-PSG studies in older men who had preserved REM sleep atonia [72] is found in conjunction with clinical symptoms suggestive of OSA. Successful treatment of the severe OSA with CPAP also controlled the violent dream-enacting behavior, as confirmed by follow up video-PSG that again found preserved REM sleep atonia, thus establishing the basis for the DEB. Such a clinical scenario highlights the diagnostic value of performing video-PSG to assess REM sleep tone and to identify possible causes of dream enactment. In cases with true overlap of OSA with RBD, not only should there be evidence of increased REM sleep muscle tone noted on PSG monitoring, but also the DEB should persist after treatment and resolution of OSA. Thus, it is mandatory to perform PSG monitoring in a case where RBD is a diagnostic consideration, to rule out OSA as a causative factor [2].

\section{Periodic limb movement disorder (PLMD)}

According to the ICSD-3, PLMD refers to a condition when PLMs disrupt sleep continuity, leading to sleep fragmentation, insomnia, non-restorative sleep, and daytime sleepiness [2]. There have been cases reported where frequent violent PLMs in sleep with DEB led to injuries to the bed partner, thus clinically simulating RBD. A recent case series analyzed such patients, where upon PSG review, it was found that the DEB consisting of flailing, kicking, and vocalization occurred after PLM-induced arousals from Non-REM sleep. REM sleep atonia was preserved. The nocturnal behavior improved substantially after initiation of dopaminergic therapy, i.e. standard therapy of RLS/PLMs. Such cases also underscore the importance of performing video-PSG studies, which are instrumental in characterizing the nature of the abnormal behaviors with dream enactment arising from sleep, and in assisting in identifying the correct diagnosis, leading to proper patient management [73].

\section{Conclusion}

A unifying concept to consider in the context of the motorbehavioral dysrhythmias associated with RBD, which were described above, involves Central Pattern Generators (CPGs), which are genetically determined neuronal aggregates in the mesencephalon, pons and spinal cord subserving innate motor behaviors, as elaborated by Tassinari et al. [74]. CPGs can produce rhythmic patterned outputs without sensory or cortical input and thus conceptually underly the complex interactions among various neurophysiological and biological rhythms in relation to RBD, described in this article. 
To conclude, the disruption of rhythmicity to varying degrees in conjunction with RBD has been manifested in abnormalities of motorbehavioral, breathing, autonomic and electrocerebral activity. As the scientific community continues on its quest to unravel the intricacies of this complex interplay of neural circuitry during sleep, more dysrhythmias associated with RBD await to be discovered.

\section{References}

1. Maquet P (2001) The role of sleep in learning and memory. Science 294: 1048-1052. [Crossref]

2. American Academy of Sleep Medicine (2014) International classification of sleep disorders. 3. Darien.

3. Siegel JM (2005) REM sleep. In: Kryger MH, Roth T, Dement WC, eds. (4th edn) Principles and Practice of Sleep Medicine. Philadelphia, PA: Elsevier Saunders, pp: 120-135.

4. Fernández-Mendoza J, Lozano B, Seijo F, Santamarta-Liébana E, Ramos-Platón MJ, et al. (2009) Evidence of subthalamic PGO-like waves during REM sleep in humans: a deep brain polysomnographic study. Sleep 32: 1117-1126. [Crossref]

5. Boeve BF (2010) REM sleep behavior disorder: Updated review of the core features, the REM sleep behavior disorder-neurodegenerative disease association, evolving concepts, controversies, and future directions. Ann N Y Acad Sci 1184: 15-54. [Crossref]

6. Irfan M, Howell MJ (2016) Rapid Eye Movement Sleep Behavior Disorder: Overview and Current Perspective. Curr Sleep Medicine Rep 2: 64.

7. Hsieh KC, Nguyen D, Siegel JM, Lai YY (2013) New pathways and data on rapid eye movement sleep behaviour disorder in a rat model. Sleep Med 14: 719-728. [Crossref]

8. Schenck CH, Boeve BF, Mahowald MW (2013) Delayed emergence of a parkinsonian disorder or dementia in $81 \%$ of older men initially diagnosed with idiopathic rapid eye movement sleep behavior disorder: a 16-year update on a previously reported series. Sleep Med 14: 744-748. [Crossref]

9. Iranzo A, Fernandez-Arcos A, Tolosa E, Serradell M, Molinuevo JL, et al. (2014) Neurodegenerative disorder risk in idiopathic REM sleep behavior disorder: study in 174 patients. PLoS One 9: e89741. [Crossref]

10. Garcia-Rill E (2009) Sleep and arousal states: reticular activating system. In: Squire LR, Bloom F, Spitzer N, Gage F, Albright T, editors. New Encyclopedia of Neuroscience. Elsevier, Oxford, England; 8: 137-143.

11. Garcia-Rill E, Kezunovic N, Hyde J, Simon C, Beck P, et al. (2013) Coherence and frequency in the reticular activating system (RAS). Sleep Med Rev 17: 227-238. [Crossref]

12. Garcia-Rill E, Kezunovic N, D'Onofrio S, Luster B, Hyde J, et al. (2014) Gamma band activity in the RAS-intracellular mechanisms. Exp Brain Res 232: 1509-1522. [Crossref]

13. Garcia-Rill E (2015) Waking and the Reticular Activating System. Academic Press, New York, pp: 330.

14. Steriade M (1999) Cellular substrates of oscillations in corticothalamic systems during states of vigilance. In Lydic R., Baghdoyan HA, editors. Handbook of Behavioral State Control. Cellular and molecular mechanisms. CRC Press, New York, pp: 327-347.

15. Wang HL, Morales M (2009) Pedunculopontine and laterodorsal tegmental nuclei contain distinct populations of cholinergic, glutamatergic and GABAergic neurons in the rat. Eur J Neurosci 29: 340-358. [Crossref]

16. Steriade M, Paré D, Datta S, Oakson G, Curro Dossi R (1990) Different cellular types in mesopontine cholinergic nuclei related to ponto-geniculo-occipital waves. $J$ Neurosci 10: 2560-2579. [Crossref]

17. Boucetta S, Cisse Y, Mainville L, Morales M, Jones BE (2014) Discharge profiles across the sleep-waking cycle of identified cholinergic, gabaergic, and glutamatergic neurons in the pontomesencephalic tegmentum of the rat. $J$ Neurosci 34: 4708-4727. [Crossref]

18. Datta S, Siwek DF (2002) Single cell activity patterns of pedunculopontine tegmentum neurons across the sleep-wake cycle in the freely moving rats. J Neurosci Res 70: 611621. [Crossref]

19. Steriade M, Curro Dossi R, Paré D, Oakson G (1991) Fast oscillations (20-40 Hz) in thalamocortical systems and their potentiation by mesopontine cholinergic nuclei in the cat. Proc Nat Acad Sci USA 88: 4396-4400. [Crossref]
20. Aserinsky E, Kleitman N (1953) Regularly occurring periods of eye motility, and concomitant phenomena, during sleep. Science 118: 273-274. [Crossref]

21. Datta S, Siwek DF, Patterson EH, Cipolloni PB (1998) Localization of pontine PGO wave generation sites and their anatomical projections in the rat. Synapse 30: 409-423. [Crossref]

22. Fraigne JJ, Torontali ZA, Snow MB, Peever JH (2015) REM Sleep at its Core - Circuits, Neurotransmitters, and Pathophysiology. Front Neurol 6: 123. [Crossref]

23. Heister DS, Hayar A, Charlesworth A, Yates C, Zhou YH, et al. (2007) Evidence for Electrical Coupling in the SubCoeruleus (SubC) Nucleus. J Neurophysiol 97: 31423147. [Crossref]

24. Garcia-Rill E, Charlesworth A, Heister D, Ye M, Hayar A (2008) The developmenta decrease in REM sleep: the role of transmitters and electrical coupling. Sleep 31: 673690. [Crossref]

25. Urbano FJ, Leznik E, Llinas R (2007) Modafinil enhances thalamocortical activity by increasing neuronal electrotonic coupling. Proc Natl Acad Sci USA 104: 12554-12559. [Crossref]

26. Jiang X, Lautermilch NJ, Watari H, Westenbroek RE, Scheuer T, et al. (2008) Modulation of $\mathrm{CaV} 2.1$ channels by $\mathrm{Ca}^{2+} /$ calmodulin-dependent protein kinase II bound to the C-terminal domain. Proc Natl Acad Sci U S A 105: 341-346. [Crossref]

27. Luster B, D'Onofrio S, Urbano F, Garcia-Rill E (2015) High-threshold $\mathrm{Ca}^{2+}$ channels behind gamma band activity in the pedunculopontine nucleus (PPN). Physiol Rep 3 . [Crossref]

28. Luster BR, Urbano FJ, Garcia-Rill E (2016) Intracellular mechanisms modulating gamma band activity in the pedunculopontine nucleus (PPN). Physiol Rep 4. [Crossref]

29. Simon C, Kezunovic N, Williams DK, Urbano FJ, Garcia-Rill E (2011) Cholinergic and glutamatergic agonists induce gamma frequency activity in dorsal subcoeruleus nucleus neurons. Amer J Physiol Cell Physiol 301: C327-C335. [Crossref]

30. Cavelli M, Castro S, Schwarzkopf N, Chase MH, Falconi A, et al. (2015) Coheren neocortical gamma oscillations decrease during REM sleep in the rat. Behav Brain Res 281: 318-325. [Crossref]

31. Torterolo P, Castro-Zaballa S, Cavelli M, Chase MH, Falconi A (2016) Neocortical 40 Hz oscillations during carbachol-induced rapid eye movement sleep and cataplexy. Eur J Neurosci 43: 580-589. [Crossref]

32. Dauvilliers Y, Jennum P, Plazzi G (2013) Rapid eye movement sleep behavior disorder and rapid eye movement sleep without atonia in narcolepsy. Sleep Med 14: 775-781. [Crossref]

33. Carter ME, Brill J, Bonnavion P, Huguenard JR, Huerta R, et al. (2012) Mechanism for Hypocretin-mediated sleep-to-wake transitions. Proc Natl Acad Sci U S A 109 E2635-2644. [Crossref]

34. Han Y, Shi Y, Xi W, Zhou R, Tan Z, Wang H, et al. (2014) Selective activation of cholinergic basal forebrain neurons induces immediate sleep-wake transitions. Curr Biol 24: 693-698. [Crossref]

35. Moruzzi G, Magoun HW (1949) Brain stem reticular formation and activation of the EEG. Electroencephalogr Clin Neurophysiol 1: 455-473. [Crossref]

36. Caillier B, Pilote S, Castonguay A, Patoine D, Ménard-Desrosiers V, et al. (2012) QRS widening and QT prolongation under buproprion: a unique cardiac electrophysiological profile. Fundam Clin Pharmacol 26: 599-608. [Crossref]

37. Sun JD, Liu Y, Yuan YH, Li J, Chen NH (2012) Gap junction dysfunction in the prefrontal cortex induces depressive-like behaviors in rats. Neuropsychopharmacology 37: 1305-1320. [Crossref]

38. Corner MA, Schenck CH (2015) Perchance to dream? Primordial motor activity patterns in vertebrates from fish to mammals: their prenatal origin, postnatal persistence during sleep, and pathological re-emergence during REM sleep behavior disorder. Neuroscience Bulletin 31: 649-662. [Crossref]

39. Roffwarg HP, Muzio JN, Dement WC (1966) Ontogenetic development of the human sleep-dream cycle. Science 152: 604-619. [Crossref]

40. Garcia-Rill E, Luster B, Mahaffey S, MacNicol M, Hyde JR, et al. (2015) Pedunculopontine Gamma Band Activity and Development. Brain Sci 5: 546-567. [Crossref]

41. Schenck CH, Mahowald MW (2002) REM sleep behavior disorder: clinical, developmental, and neuroscience perspectives 16 years after its formal identification in SLEEP. Sleep 25: 120-138. [Crossref] 
42. Fantini ML, Michaud M, Gosselin N, Lavigne G, Montplaisir J (2002) Periodic leg movements in REM sleep behavior disorder and related autonomic and EEG activation. Neurology 59: 1889-1894. [Crossref]

43. Lai Y-Y, Hsieh K-C, Nguyen D, Peever J, Siegel JM (2008) Neurotoxic lesions at the mesopontine junction change sleep time and muscle activity during sleep: an animal model of motor disorders in sleep. Neuroscience 154: 431-443. [Crossref]

44. Iranzo A, Tolosa E, Gelpi, E (2013) Neurodegenerative disease status and post-mortem pathology in idiopathic rapid-eye-movement sleep behaviour disorder: an observational cohort study. Lancet Neurol 12: 443-453. [Crossref]

45. Schenck CH, Bundlie SR, Mahowald MW (1996) Delayed emergence of a parkinsonian disorder in $38 \%$ of 29 older men initially diagnosed with idiopathic rapid eye movement sleep behavior disorder. Neurology 46: 388-393. [Crossref]

46. Miyamoto M, Miyamoto T, Kubo J, Yokota N, Hirata K, et al. (2000) Brainstem function in rapid eye movement sleep behavior disorder: the evaluation of brainstem function by proton MR spectroscopy (1H-MRS). Psychiatry Clin Neurosci 54: 350351. [Crossref]

47. Abele M, Burk K, Andres F, Topka H, Laccone F, et al. (1997) Autosomal dominant cerebellar ataxia type I. Nerve conduction and evoked potential studies in families with SCA1, SCA2 and SCA3. Brain 120: 2141-2148. [Crossref]

48. Friedman JH (2002) Presumed rapid eye movement behavior disorder in MachadoJoseph disease (spinocerebellar ataxia type 3). Mov Disord 17: 1350-1353. [Crossref]

49. Iranzo A, Munoz E, Santamaria J, Vilaseca I, Mila M, et al. (2003) REM sleep behavior disorder and vocal cord paralysis in Machado-Joseph disease. Mov Disord 18: 11791183. [Crossref]

50. Chi NF, Shiao GM, Ku HL, Soong BW (2013) Sleep disruption in spinocerebella ataxia type 3: a genetic and polysomnographic study. J Chin Med Assoc 76: 25-30. [Crossref]

51. Pedroso JL, Braga-Neto P, Martinez AR, Martins CR Junior, Rezende Filho FM, et al (2016) Sleep disorders in Machado-Joseph disease. Curr Opin Psychiatry 29:402-408. [Crossref]

52. Abe S, Gagnon J-F, Montplaisir JY, Postuma RB, Rompré PH, et al. (2013) Sleep bruxism and oromandibular myoclonus in rapid eye movement sleep behavior disorder: a preliminary report. Sleep Med 14: 1024-1030. [Crossref]

53. Tachibana N, Yamanaka K, Kaji R, Nagamine T, Watatani K, et al. (1994) Sleep bruxism as a manifestation of subclinical rapid eye movement sleep behavior disorder. Sleep 17: 555-558. [Crossref]

54. Schenck CH, Mahowald MW (2008) Subclinical REM sleep behavior disorder and its clinical and research implications. Sleep 31: 1627. [Crossref]

55. Martynowicz H, Wieczorek T, Michalek M (2017) Sexsomnia related to severe bruxism? A case report. Sleep Med. Abstract(in press).

56. Manni R, Terzaghi M (2007) Rhythmic movements in idiopathic REM sleep behavior disorder. Mov Disord 22: 1797-1800. [Crossref]

57. Xu Z, Anderson KN, Shneerson JM (2009) Association of idiopathic rapid eye movement sleep behavior disorder in an adult with persistent, childhood onset rhythmic movement disorder. J Clin Sleep Med 5: 374-375. [Crossref]

58. Manni R, Terzaghi M, Zambrelli E (2007) REM sleep behaviour disorder in elderly subjects with epilepsy: frequency and clinical aspects of the comorbidity. Epilepsy Res 77: 128-133. [Crossref]
59. Manni R, Terzaghi M, Zambrelli E, Pacchetti C (2006) Interictal, potentially misleading, epileptiform EEG abnormalities in REM sleep behavior disorder. Sleep 29: 934-937. [Crossref]

60. Andrillon T, Nir Y, Staba RJ, Ferrarelli F, Cirelli C, et al. (2011) Sleep spindles in humans: insights from intracranial EEG and unit recordings. $J$ Neurosci 31: 1782117834. [Crossref]

61. O'Reilly C1,2,3, Godin I1,4, et al. (2015) REM sleep behaviour disorder is associated with lower fast and higher slow sleep spindle densities. J Sleep Res 24: 593-601. [Crossref]

62. Christensen JA, Kempfner J, Zoetmulder M, Leonthin HL, Arvastson L, et al. (2014) Decreased sleep spindle density in patients with idiopathic REM sleep behavior disorder and patients with Parkinson's disease. Clin Neurophysiol 125: 512-519. [Crossref]

63. Ozaki A, Nishida M, Koyama K, Ishikawa K, Nishikawa T (2012) Donepezilinduced sleep spindle in a patient with dementia with Lewy bodies: a case report. Psychogeriatrics 12: 255-258. [Crossref]

64. Postuma RB, Gagnon JF, Pelletier A, Montplaisir J (2013) Prodromal autonomic symptoms and signs in Parkinson's disease and dementia with Lewy bodies. Mov Disord 28: 597-604. [Crossref]

65. Ferini-Strambi L, Oertel W, Dauvilliers Y, Postuma RB, Marelli S, et al. (2014) Autonomic symptoms in idiopathic REM behavior disorder: a multicentre case-control study. J Neurol 261: 1112-1118. [Crossref]

66. Fereshtehnejad SM, Montplaisir JY, Pelletier A, Gagnon JF, Berg D, et al. (2017) Validation of the MDS research criteria for prodromal Parkinson's disease: Longitudinal assessment in a REM sleep behavior disorder (RBD) cohort. Mov Disord 32: 865-873. [Crossref]

67. Kaufmann H, Norcliffe-Kaufmann L, Palma JA, Biaggioni I, Low PA, et al. (2017) Natural history of pure autonomic failure: A United States prospective cohort. Ann Neurol 81: 287-297. [Crossref]

68. Schenck CH, Milner DM, Hurwitz TD, Bundlie SR, Mahowald MW (1989) A polysomnographic and clinical report on sleep-related injury in 100 adult patients. $\mathrm{Am}$ J Psychiatry 146: 1166-1173. [Crossref]

69. Schenck CH (2005) Paradox Lost: Midnight in the Battleground of Sleep and Dreams. Minneapolis, MN: Extreme-Nights, LLC.

70. Pillmann F (2009) Complex dream-enacting behavior in sleepwalking. Psychosom Med 71: 231-234. [Crossref]

71. Haridi M, Weyn Banningh S, Clé M, et al. (2017) Is there a common moto dysregulation in sleepwalking and REM sleep behaviour disorder? J Sleep Res 26: 614622. [Crossref]

72. Iranzo A, Santamaría J (2002) Severe Obstructive Sleep Apnea/Hypopnea Mimicking REM Sleep Behavior Disorder. Sleep 28: 203-206. [Crossref]

73. Gaig C, Iranzo A, Pujol M, Perez H, Santamaria J (2017) Periodic Limb Movements During Sleep Mimicking REM Sleep Behavior Disorder: A New Form of Periodic Limb Movement Disorder. Sleep. [Crossref]

74. Tassinari CA, Rubboli G, Gardella E, Cantalupo G, Calandra-Buonaura G, et al. (2005) Central pattern generators for a common semiology in fronto-limbic seizures and in parasomnias. A neuroethologic approach. Neurol Sci 26: s225-s232. [Crossref]

Copyright: ( 02017 Irfan M. This is an open-access article distributed under the terms of the Creative Commons Attribution License, which permits unrestricted use, distribution, and reproduction in any medium, provided the original author and source are credited. 